\title{
HOW MUCH IS YOUR DIET? \\ (ESTIMATION ABOUT PRICES OF “TRADITIONAL HUNGARIAN", DIABETIC, LOW ENERGY DIETS, AND RELATED LIFE-STYLE EXPENSES)
}

\author{
G. IsKI ${ }^{a}$, L. BIRób ${ }^{\text {, T. UNGVÁRI }}{ }^{\mathrm{a}}$ and I. RURIK ${ }^{\mathrm{a} *}$ \\ ${ }^{a}$ Department of Family and Occupational Medicine, Faculty of Public Health, University of Debrecen, \\ H-4032 Debrecen. Móricz Zsigmond krt. 22. Hungary \\ ${ }^{b}$ NutriComp Ltd. H-1091 Budapest, Üllői út 179. Hungary
}

(Received: 1 June 2015; accepted: 30 November 2015)

Nutrition and lifestyle-related diseases are some of the leading morbidities among the Hungarian population. People who want to lose weight often complain that healthy diet is expensive.

Our aim was to quantify the costs of three different types of diet for a three-day period. We compared "traditional Hungarian", low energy, and diabetic diets, considering both energy content and expenses related to lifestyle.

According to our estimation: diabetic (including medication) and "traditional" Hungarian diets were the most expensive. Low energy diet proved to be the most cost-effective despite the extra expenditures of higher physical activity.

Keywords: costs, diabetic diet, expenses, Hungarian diet, low energy diet

Research has clearly indicated that diet plays an important role in the prevention of obesity and related conditions like diabetes. Nutrition has come to the fore as one of the major modifiable determinants of chronic diseases. Dietary and lifestyle patterns can produce substantial gains in the population's health (STORY et al., 2007).

Proper diet can support the required lifestyle changes, treatments, and medications as well. A healthy diet should contain macro -and micro -nutrients with vitamins, minerals, and trace elements in sufficient quantities. The number of scientific evidence emphasizing the role of key nutrients in full health, vitality, and longevity is increasing (USDA, 2010).

Diet and lifestyle-related diseases (cardiovascular diseases, cancer, diabetes, and obesity) are the leading diseases of the Hungarian population and are responsible for the majority of early mortality (KSH, 2012).

Special diets are frequently used by obese persons to reduce body weight, by diabetics to provide the proper ratio of macronutrients, and often to reduce energy intake as well (LEHOTA et al., 2014).

Medical Nutritional Therapy can offer a special focus to the needs of diabetic and obese patients (VetTer et al., 2014). Organic diet has become more popular in the last decade based

\footnotetext{
* To whom correspondence should be addressed. Phone: +36 5225 52-52; fax: 365225 52-53; e-mail: Rurik.Imre@sph.unideb.hu
} 
on the natural way of production (and ripening) without using chemicals in the agricultural processes.

The so-called "traditional Hungarian diet" is rich in fats and added salt and requires specific preparation cooking/baking processes. Many of these foods and dishes are considered as unhealthy "typical flavours" (BIRÓ, 2007).

"Very low carbohydrate" and ,very low fat" diets recommended for weight reduction usually contain higher proportion of protein beside lower carbohydrates and fats.

Those who are obese or want to lose weight often complain that a healthy diet is too expensive. A significant proportion of obese people live in socially disadvantaged circumstances, making the cost/price of food an even more important issue. Food costs are frequently cited as a reason for failure to address eating behaviours in practice; this is a perceived barrier rather than a real one. Other factors, such as taste, time scarcity, and cooking skills of the family, impact upon family food-purchasing choices. In the present economic climate, it is possible that the current cost of healthier food might rise at a higher trajectory than low-cost, energy-dense food types (BANKS et al., 2012).

\section{Materials and methods}

\subsection{Diets}

Our objective was to assess and compare three different diets in terms of expenses. The costs and energy contents of three different diets were estimated: I. "traditional Hungarian", II. "low energy", and III. "diabetic", subjected to a 3-day period and compared. Energy content, medications, and expenses related to lifestyle were considered as well.

\subsection{Methods of analysis}

The energy contents of three different 3-day sample diets were compared using a specific software, NutriComp® (Nutricomp LTD, Budapest). Besides the prices of food and dishes, the average expenses of physical activities and medications were also considered. Expenditures were determined by the average Hungarian retail and wholesale prices, in the year of 2013, based on the data published by the Hungarian Central Statistical Office (KSH, 2013).

Ethical permission was not required to this study.

\section{Results and discussion}

I. "Traditional Hungarian", known as generally consumed or habitual diet (Table 1): The average energy content of a traditional Hungarian diet per day is approximately 3000 calories. This includes many foods and ingredients that professionals do not recommend because of their high calorie content and unhealthy composition.

The daily average cost of the "traditional Hungarian" 3-day model diet was 2211 HUF. People who prefer this diet are usually overweight and usually drink sweetened soft drinks (LUGASI et al., 2010; SARKADI NAGY et al., 2012). If we consider this extra consumption, the daily cost could be as much as $2800 \mathrm{HUF} /$ day. 
ISKI et al.: HOW MUCH IS YOUR DIET?

Table 1. „Traditional” Hungarian diet

\begin{tabular}{|c|c|c|c|c|c|}
\hline & & $\begin{array}{c}\text { Price } \\
(\mathrm{HUF}) / 100 \mathrm{~g}\end{array}$ & $\begin{array}{c}\text { Energy } \\
\text { (Kcal) } / 100 \mathrm{~g}\end{array}$ & $\begin{array}{l}\text { Consumed } \\
\text { dose }(\mathrm{g}) \text { If } \\
\text { other* }\end{array}$ & $\begin{array}{c}\text { Consumed } \\
\text { food cost } \\
\text { (HUF) }\end{array}$ \\
\hline \multirow[t]{2}{*}{ 1. day } & chocolate milk & 24 & 64 & $250 \mathrm{ml}^{*}$ & 60 \\
\hline & butter & 128 & 727 & 10 & 13 \\
\hline \multirow{2}{*}{$\begin{array}{l}\text { Breakfast } \\
(594 \text { Kcal })\end{array}$} & jam/marmalade & 166 & 263 & 60 & 100 \\
\hline & milk-loaf & 99 & 403 & 50 & 50 \\
\hline \multirow{3}{*}{$\begin{array}{l}\text { Snack/ } \\
\text { elevenses } \\
(670 \mathrm{Kcal})\end{array}$} & butter & 128 & 727 & 10 & 13 \\
\hline & crescent & 40 & 616 & 100 & 40 \\
\hline & ham & 239 & 160 & 50 & 120 \\
\hline \multirow{2}{*}{$\begin{array}{l}\text { Lunch } \\
\text { (1196 Kcal) }\end{array}$} & chicken soup with angel hair & 101 & 87 & 400 & 404 \\
\hline & pig stew, noodles & 186 & 212 & 400 & 744 \\
\hline \multirow{3}{*}{$\begin{array}{l}\text { Dinner } \\
\text { (533 Kcal) }\end{array}$} & sausage & 316 & 293 & 125 & 395 \\
\hline & bread & 28 & 259 & 60 & 17 \\
\hline & cucumber & 50 & 12 & 100 & 50 \\
\hline Total & & & & (2993 Kcal) & 2006 \\
\hline \multirow{2}{*}{ 2. day } & bread & 28 & 259 & 60 & 17 \\
\hline & bacon & 130 & 447 & 100 & 130 \\
\hline \multirow[t]{2}{*}{$\begin{array}{l}\text { Breakfast } \\
(774 \text { Kcal) }\end{array}$} & cucumber & 50 & 12 & 100 & 50 \\
\hline & chocolate milk & 25 & 64 & $250 \mathrm{ml}^{*}$ & 63 \\
\hline \multirow{3}{*}{$\begin{array}{l}\text { Snack/ } \\
\text { elevenses } \\
(712 \mathrm{Kcal})\end{array}$} & pudding & 54 & 70 & 200 & 108 \\
\hline & waffles & 99 & 415 & 100 & 99 \\
\hline & jam/marmalade & 166 & 263 & 60 & 100 \\
\hline \multirow{2}{*}{$\begin{array}{l}\text { Lunch } \\
\text { (922 Kcal) }\end{array}$} & meatball and vegetable soup & 120 & 49 & 400 & 480 \\
\hline & pork greaves & 195 & 182 & 400 & 780 \\
\hline \multirow{3}{*}{$\begin{array}{l}\text { Snack } \\
(219 \text { Kcal }) \\
\text { Dinner } \\
(226 \text { Kcal }) \\
\text { Total }\end{array}$} & $\begin{array}{l}\text { chestnut puree with whipped } \\
\text { cream }\end{array}$ & 250 & 219 & 100 & 250 \\
\hline & pizza & 300 & 226 & 100 & 300 \\
\hline & & & & $(2854 \mathrm{Kcal})$ & 2377 \\
\hline \multirow{2}{*}{ 3. day } & bread & 28 & 259 & 60 & 17 \\
\hline & sausage & 316 & 293 & 125 & 395 \\
\hline \multirow[t]{2}{*}{$\begin{array}{l}\text { Breakfast } \\
(543 \text { Kcal) }\end{array}$} & tomato & 100 & 22 & 100 & 100 \\
\hline & butter & 128 & 727 & 10 & 13 \\
\hline \multirow{3}{*}{$\begin{array}{l}\text { Snack/ } \\
\text { elevenses } \\
(494 \text { Kcal })\end{array}$} & bread & 28 & 259 & 60 & 17 \\
\hline & cheese & 170 & 372 & 50 & 85 \\
\hline & ham & 239 & 160 & 50 & 120 \\
\hline \multirow{2}{*}{$\begin{array}{l}\text { Lunch } \\
\text { (1303 Kcal) }\end{array}$} & bean soup & 120 & 70 & 400 & 480 \\
\hline & $\begin{array}{l}\text { garlic, roasted flitch rice with } \\
\text { peas }\end{array}$ & 204 & 256 & 400 & 816 \\
\hline $\begin{array}{l}\text { Snack } \\
(216 \text { Kcal })\end{array}$ & pancakes with jam & 160 & 270 & 80 & 128 \\
\hline $\begin{array}{l}\text { Dinner } \\
(165 \mathrm{Kcal})\end{array}$ & egg & 80 & 165 & 2 pieces* & 80 \\
\hline Total & & & & (2721 Kcal) & 2251 \\
\hline Mean & & & & & 2211 \\
\hline
\end{tabular}

Acta Alimentaria 45, 2016 
II. Low energy diet (Table 2): Estimating the costs of healthy (physically active) lifestyle in Hungary, expenses were the lowest with this type of diet. In a low energy diet the recommended energy intake was 1500-2000 calories/day for a man with average body size and physical activity. The daily cost of meal was 1870 HUF. People who would like to lose weight usually drink water, so the consumed fluid during the day from non-carbonated mineral water was $200 \mathrm{HUF} / 2$ litre. In addition, (multi)vitamin supplementation, (approximately $2000 \mathrm{HUF} / 30$ pieces) cost $67 \mathrm{HUF}$ a day, amounting to a total of $2137 \mathrm{HUF}$. Being an essential element of healthy lifestyle, regular exercise in a fitness club or gym was estimated to cost $10000 \mathrm{HUF}$ a month; further sport-related cost/day was $334 \mathrm{HUF}$, making the daily total expenditure $2471 \mathrm{HUF}$.

\begin{tabular}{|c|c|c|c|c|c|}
\hline & & $\begin{array}{c}\text { Price } \\
(\mathrm{HUF}) / 100 \mathrm{~g}\end{array}$ & $\begin{array}{c}\text { Energy } \\
\text { (Kcal) } / 100 \mathrm{~g}\end{array}$ & $\begin{array}{c}\text { Consumed } \\
\text { dose }(\mathrm{g}) \\
\text { If other* } \\
\end{array}$ & $\begin{array}{c}\text { Consumed } \\
\text { food cost } \\
\text { (HUF) }\end{array}$ \\
\hline 1. day & fruit tea & 1400 & 0 & 1 & 14 \\
\hline & Trappist cheese & 170 & 340 & 100 & 170 \\
\hline \multirow{2}{*}{$\begin{array}{l}\text { Breakfast } \\
(433 \text { Kcal) }\end{array}$} & bread & 28 & 259 & 30 & 9 \\
\hline & radish & 300 & 15 & 100 & 300 \\
\hline \multirow{3}{*}{$\begin{array}{l}\text { Snack/ } \\
\text { elevenses } \\
(149 \text { Kcal })\end{array}$} & puffed rice & 149 & 354 & 20 & 30 \\
\hline & buttercream & 180 & 380 & 10 & 18 \\
\hline & pepper & 50 & 20 & 200 & 100 \\
\hline \multirow{4}{*}{$\begin{array}{l}\text { Lunch } \\
(675 \text { Kcal) } \\
\text { Snack } \\
\text { ( } 70 \text { Kcal) } \\
\text { Dinner } \\
(455 \text { Kcal) }\end{array}$} & $\begin{array}{l}\text { currant cream soup, cauli- } \\
\text { flower casserole, pork }\end{array}$ & 230 & 169 & 400 & 920 \\
\hline & apple & 28 & 35 & 200 & 56 \\
\hline & frankfurter & 100 & 105 & 60 & 100 \\
\hline & greenpea stew & 100 & 392 & 400 & 400 \\
\hline Total & & & & (1782 Kcal) & 2117 \\
\hline \multirow[t]{2}{*}{ 2. day } & orange juice & 42 & 59 & $200 \mathrm{ml}^{*}$ & 84 \\
\hline & egg & 40 & 143 & 1 pieces* & 20 \\
\hline \multirow{2}{*}{$\begin{array}{l}\text { Breakfast } \\
(486 \mathrm{Kcal})\end{array}$} & bun & 42 & 273 & 100 & 42 \\
\hline & cucumber & 50 & 12 & 100 & 50 \\
\hline $\begin{array}{l}\text { Snack/ } \\
\text { elevenses } \\
(170 \text { Kcal })\end{array}$ & fruit salad & 100 & 170 & 100 & 100 \\
\hline $\begin{array}{l}\text { Lunch } \\
\text { (977 Kcal) }\end{array}$ & $\begin{array}{l}\text { potato soup with } \\
\text { vegetables, fried liver, } \\
\text { greenbean stew, bread }\end{array}$ & 230 & 244 & 400 & 920 \\
\hline $\begin{array}{l}\text { Snack } \\
(60 \mathrm{Kcal})\end{array}$ & pumpkin & 12 & 30 & 200 & 24 \\
\hline \multirow[t]{4}{*}{$\begin{array}{l}\text { Dinner } \\
(428 \mathrm{Kcal})\end{array}$} & ham & 221 & 157 & 100 & 221 \\
\hline & cow's milk & 25 & 88 & $200 \mathrm{ml}^{*}$ & 50 \\
\hline & bread & 28 & 259 & 30 & 9 \\
\hline & salad & 85 & 17 & 100 & 85 \\
\hline Total & & & & (2121 Kcal) & 1605 \\
\hline
\end{tabular}




\begin{tabular}{|c|c|c|c|c|c|}
\hline & & $\begin{array}{c}\text { Price } \\
(\mathrm{HUF}) / 100 \mathrm{~g}\end{array}$ & $\begin{array}{c}\text { Energy } \\
\text { (Kcal) } / 100 \mathrm{~g}\end{array}$ & $\begin{array}{l}\text { Consumed } \\
\text { dose }(\mathrm{g}) \\
\text { If other* }\end{array}$ & $\begin{array}{c}\text { Consumed } \\
\text { food cost } \\
\text { (HUF) }\end{array}$ \\
\hline 3. day & $\begin{array}{l}\text { cow's milk } \\
\text { poultry frankfurter }\end{array}$ & $\begin{array}{c}25 \\
100\end{array}$ & $\begin{array}{c}88 \\
105\end{array}$ & $\begin{array}{c}200 \mathrm{ml}^{*} \\
100\end{array}$ & $\begin{array}{c}50 \\
100\end{array}$ \\
\hline $\begin{array}{l}\text { Breakfast } \\
\text { (399 Kcal) }\end{array}$ & bread & 28 & 259 & 30 & 9 \\
\hline & pepper & 50 & 20 & 200 & 100 \\
\hline $\begin{array}{l}\text { Snack/ } \\
\text { elevenses } \\
(157 \text { Kcal })\end{array}$ & grape & 70 & 81 & 150 & 105 \\
\hline $\begin{array}{l}\text { Lunch } \\
\text { (764 Kcal) }\end{array}$ & $\begin{array}{l}\text { cracker bread } \\
\text { chicken soup, pasta with } \\
\text { cabbage }\end{array}$ & 220 & 350 & 400 & 920 \\
\hline $\begin{array}{l}\text { Snack } \\
(141 \text { Kcal })\end{array}$ & kefír & 50 & 65 & $150 \mathrm{ml}^{*}$ & 75 \\
\hline & wholemeal biscuit & 150 & 423 & 10 & 15 \\
\hline $\begin{array}{l}\text { Dinner } \\
(175 \mathrm{Kcal})\end{array}$ & ham & 239 & 160 & 50 & 120 \\
\hline & asparagus & 250 & 16 & 100 & 250 \\
\hline & tomato & 50 & 22 & 200 & 100 \\
\hline & cracker bread & 220 & 350 & 10 & 22 \\
\hline Total & & & & (1635 Kcal) & 1888 \\
\hline Mean & & & & & 1870 \\
\hline
\end{tabular}

III. Diabetic diet as a part of the Medical Nutrition Therapy (Table 3): A diabetic man with average anthropometric parameters is expected to consume 1600-1800 calories per day. The daily price of this specific diet was calculated to cost 2054 HUF (FöVÉNYI \& GYURCSÁNÉ, 2014). Patients need sugar-free drinks, which usually cost more than mineral water. In comparison, mineral water costs $200 \mathrm{HUF} / 2$ litre, sugar-free soft drinks are sold for 442 HUF/litre, while sugar-free syrup is $403 \mathrm{HUF} / 0.5$ litre. Even if following the recommendations of Medical Nutritional Therapy strictly, most of the patients need medications as well. The monthly average cost of oral antidiabetic drugs falls in the range of 500-4000 HUF, i.e. (17-133 HUF/day. Diabetic patients are also advised to exercise at least three times a week, and the same expenses are considered as calculated above (334 HUF). Total daily cost amounts to 2892 HUF (Table 3).

\begin{tabular}{|c|c|c|c|c|c|}
\hline & & $\begin{array}{c}\text { Price } \\
(\mathrm{HUF}) / 100 \mathrm{~g}\end{array}$ & $\begin{array}{c}\text { Energy } \\
(\text { Kcal }) / 100 \mathrm{~g}\end{array}$ & $\begin{array}{c}\text { Consumed } \\
\text { dose }(\mathrm{g}) \\
\text { If other* }\end{array}$ & $\begin{array}{c}\text { Consumed } \\
\text { food cost } \\
\text { (HUF) }\end{array}$ \\
\hline & freshly squeezed orange & & & & \\
\hline \multirow[t]{3}{*}{ 1. day } & juice & 42 & 50 & $200 \mathrm{ml}^{*}$ & 84 \\
\hline & cornflakes & 120 & 423 & 30 & 36 \\
\hline & milk & 24 & 53 & $200 \mathrm{ml}^{*}$ & 48 \\
\hline \multicolumn{6}{|l|}{ Breakfast } \\
\hline (575 Kcal) & wholegrain bread & 40 & 256 & 50 & 20 \\
\hline & plant origin margarine & 128 & 325 & 3.5 & 45 \\
\hline \multicolumn{6}{|l|}{$\begin{array}{l}\text { Snack/ } \\
\text { elevences }\end{array}$} \\
\hline (118 Kcal) & apple & 28 & 59 & 200 & 56 \\
\hline
\end{tabular}


Table 3 continued

\begin{tabular}{|c|c|c|c|c|c|}
\hline & & $\begin{array}{c}\text { Price } \\
(\mathrm{HUF}) / 100 \mathrm{~g}\end{array}$ & $\begin{array}{c}\text { Energy } \\
(\mathrm{Kcal}) / 100 \mathrm{~g}\end{array}$ & $\begin{array}{c}\text { Consumed } \\
\text { dose }(\mathrm{g}) \\
\text { If other* }\end{array}$ & $\begin{array}{c}\text { Consumed } \\
\text { food cost } \\
\text { (HUF) } \\
\end{array}$ \\
\hline $\begin{array}{l}\text { Lunch } \\
\text { ( } 746 \text { Kcal) }\end{array}$ & $\begin{array}{l}\text { steamed seafish } \\
\text { with wholegrain bread, plant } \\
\text { origin margarine, butter- } \\
\text { bean, } \\
\text { grilled tomatoes, broccoli }\end{array}$ & 322 & 187 & 400 & 1288 \\
\hline Snack & wholegrain bread & 40 & 256 & 30 & 12 \\
\hline $\begin{array}{l}(154 \mathrm{Kcal}) \\
\text { Dinner }\end{array}$ & $\begin{array}{l}\text { cottage cheese } \\
\text { fibrous fruit juice or }\end{array}$ & 143 & 76 & 100 & 143 \\
\hline $\begin{array}{l}(80 \mathrm{Kcal}) \\
\text { Extra dinner }\end{array}$ & home-cooked soup & 42 & 70 & 115 & 48 \\
\hline $\begin{array}{l}(106 \mathrm{Kcal}) \\
\text { Total }\end{array}$ & pear & 40 & 53 & $\begin{array}{c}200 \\
(1779 \mathrm{Kcal}) \\
\end{array}$ & $\begin{array}{c}80 \\
1860 \\
\end{array}$ \\
\hline 2. day & tomato juice & 30 & 20 & 115 & 35 \\
\hline $\begin{array}{l}\text { Breakfast } \\
(175 \mathrm{Kcal}) \\
\text { Snack/ } \\
\text { elevenses }\end{array}$ & $\begin{array}{l}\text { yogurt } \\
\text { puffed rice }\end{array}$ & 127 & 354 & 125 & 159 \\
\hline (89 Kcal) & $\begin{array}{l}\text { grated apple or pear } \\
\text { cold roast beef, } \\
\text { baked potato greased with }\end{array}$ & 40 & 59 & 150 & 60 \\
\hline $\begin{array}{l}\text { Lunch } \\
\text { ( } 600 \mathrm{Kcal})\end{array}$ & $\begin{array}{l}\text { plant origin margarine, } \\
\text { skimmed milk, } \\
\text { mixed vegetable salad, } \\
\text { orange }\end{array}$ & 322 & 150 & 400 & 1288 \\
\hline Snack & crispbread & 289 & 36 & 2 slices* & 29 \\
\hline (199 Kcal) & egg & 80 & 165 & 2 pieces* & 80 \\
\hline $\begin{array}{l}\text { Dinner } \\
(230 \mathrm{Kcal}) \\
\text { Extra dinner }\end{array}$ & $\begin{array}{l}\text { brown rice, } \\
\text { natural chicken }\end{array}$ & 250 & 200 & 115 & 288 \\
\hline (208 Kcal) & orange & 38 & 41 & 200 & 76 \\
\hline & sultanas & 178 & 282 & 4 pieces* & 80 \\
\hline Total & & & & (1501 Kcal) & 2125 \\
\hline 3. day & $\begin{array}{l}\text { grapefruit juice } \\
\text { egg }\end{array}$ & $\begin{array}{l}48 \\
80\end{array}$ & $\begin{array}{c}52 \\
165\end{array}$ & $\begin{array}{c}115 \\
2 \text { pieces* }\end{array}$ & $\begin{array}{l}55 \\
80\end{array}$ \\
\hline $\begin{array}{l}\text { Breakfast } \\
\text { (424 Kcal) }\end{array}$ & $\begin{array}{l}\text { wholegrain bread } \\
\text { butter }\end{array}$ & $\begin{array}{c}40 \\
128\end{array}$ & $\begin{array}{l}256 \\
727\end{array}$ & $\begin{array}{l}50 \\
10\end{array}$ & $\begin{array}{l}20 \\
13\end{array}$ \\
\hline $\begin{array}{l}\text { Snack/ } \\
\text { elevenses } \\
(173 \text { Kcal })\end{array}$ & $\begin{array}{l}\text { wholegrain bread } \\
\text { lean ham }\end{array}$ & $\begin{array}{c}40 \\
239\end{array}$ & $\begin{array}{l}256 \\
157\end{array}$ & $\begin{array}{l}50 \\
30\end{array}$ & $\begin{array}{l}20 \\
72\end{array}$ \\
\hline $\begin{array}{l}\text { Lunch } \\
\text { (824 Kcal) }\end{array}$ & $\begin{array}{l}\text { whole wheat bread greased } \\
\text { with plant origin margarine, } \\
\text { seafish (fresh or canned } \\
\text { without oil), sliced } \\
\text { cucumber, } \\
\text { apple or pear }\end{array}$ & 322 & 206 & 400 & 1288 \\
\hline $\begin{array}{l}\text { Snack } \\
(126 \mathrm{Kcal}) \\
\text { Dinner }\end{array}$ & sultanas & 178 & 282 & 4 pieces* & 80 \\
\hline $\begin{array}{l}(150 \mathrm{Kcal}) \\
\text { Extra dinner }\end{array}$ & vegetable or salad & 333 & 100 & 150 & 500 \\
\hline $\begin{array}{l}(68 \mathrm{Kcal}) \\
\text { Total } \\
\text { Mean }\end{array}$ & grapefruit & 37 & 52 & $\begin{array}{c}130 \\
(1765 \text { Kcal })\end{array}$ & $\begin{aligned} & 48 \\
& 2176 \\
& 2054\end{aligned}$ \\
\hline
\end{tabular}




\section{Conclusions}

As far as the financial rank list is concerned, diabetic diet turned out to be the most expensive. As excess weight is predominantly present years before the onset of diabetes, and also it plays a crucial role in triggering the disease, proper nutritional habits can prevent (or delay) the onset of disease. In Hungary, 5-6\% of the total adult population is estimated to suffer from diabetes (KSH, 2014). At least $75 \%$ of them also have excess body weight. Weight reduction is essential for obese diabetic patients. The sooner this is achieved, the more significant improvement can be observed in the individual's life expectancy and therapeutic outcomes (KéKEs \& KISS, 2014). In favourable cases diabetes may even regress or at least become easier to manage. Therefore, dietary treatment and proper medication are the main tools of management for obese diabetics.

Energy-rich, traditional Hungarian dishes constitute the second most costly diet, which, unfortunately, is combined with a basically sedentary lifestyle. Poor eating habits are inevitably passed on to the children as a model to follow.

Low energy diet and physically active lifestyle appear to be the most cost-effective combination. Obesity, the development of diabetes and certain other diseases caused by inappropriate nutrition and lifestyle are preventable (MóczÁR \& RURIK, 2015), improved vitality being an extra bonus. According to the official estimations, approximately a monthly income of 120000 HUF per capita is deemed necessary to keep up the average standard of living in Hungarian households; 70000 HUF and 215000 HUF representing minimum and good levels, respectively (KSH, 2013). According to our calculations, healthy lifestyle costs approximately 75000 HUF.

In the United States, where the prevalence of obesity is the highest, low energy diet is less acceptable among low-income families. Based on the researchers' findings, 2000-calorie diet would just cost $\$ 3.52$ a day if it consisted of junk food, compared with $\$ 36.32$ a day for a diet of low energy dense foods (PARKer-Pope, 2007). In the United Kingdom and in Germany, the cost of every lost kilogram from body weight was calculated as 122 USD (Fuller et al., 2013). Another US study examined the price elasticity of demand of sugarsweetened beverages, fast food restaurants, and fruit and vegetables. The cheaper prices were associated with lower bodyweight, suggesting that reducing the cost of fruit and vegetables seems to be effective in reducing obesity (Powell et al., 2013).

Although this quasi arbitrary comparison considering the findings above and some previous studies proved that expenses related to nutritional habits and lifestyle practice could modify the health status of individuals even to improve or deteriorate, while individual differences could be wide and hardly comparable. Our study underlines the importance of healthy food choices and lifestyle, which could be the best saving of expenses for the future.

Authors thank to Jusztina N. Jánossyné for the English language corrections.

Conflicts of interest: all authors declare no conflicts of interests and no financial support regarding this study, its data collection and analysis. IR received a grant to finance another, obesity-related scientific research (IGEN-TÁMOP 4.22.A-II/I KON-2012-003). There was no other financial support or accepted grant from any bodies or organizations.

Actual exchange rates at the time of survey (2013):
$1 \mathrm{HUF}=0.0032$ EUR
$1 \mathrm{EUR}=315 \mathrm{HUF}$
$1 \mathrm{HUF}=0.0039 \mathrm{USD}$
$1 \mathrm{USD}=255 \mathrm{HUF}$ 


\section{References}

Banks, J., Williams, J., Cumberlidge, T., Cimonetti, T., Sharp, D.J. \& Shield, J.P.H. (2012): Is healthy eating for obese children necessarily more costly for families? Brit. J. Gen. Pract., 62, 16-17.

Biró, Gy. (2007): Public health nutrition in Hungary - Facts and hopes. Hung. Med. J., 1(1), 7-12.

FövÉNYI, J. \& GyuRCSÁNÉ, K.I. (2014): GI-diéta (GI-diet). SpringMed., Budapest, pp. 96-105.

Fuller, N.R., Colagiuri, S., Schofield, D., Olson, A.D., Shrestha, R., Holzapfel, C., Wolfenstetter, S.B., Holle, R., Ahern, A.L., Hauner, H., JebB, S.A. \& CATERson, I.D. (2013): A within-trial cost-effectiveness analysis of primary care referral to a commercial provider for weight loss treatment, relative to standard care - an international randomised controlled trial. Int. J. Obesity, 37(6), 828-834.

KéKES, E. \& KIsS, I. (2014): Vérnyomás-variabilitás mérése és klinikai értéke (Measurement of blood pressure variability and its clinical value). Orv. Hetil., 155, 1661-1672.

KSH (2012): Available at https://www.ksh.hu/docs/hun/xstadat/xstadat_eves/i_wnh001.html (last accessed: 07 Jul 2015)

KSH (2013): Available at https://www.ksh.hu/docs/hun/xstadat/xstadat_eves/i_qsf003b.html (last accessed: 07 Jul 2015)

KSH (2013): Available at https://www.ksh.hu/docs/hun/xstadat/xstadat eves/i zhc012.html (last accessed: 07 Jul 2015)

KSH (2014): Available at http://www.ksh.hu/docs/hun/xstadat/xstadat_eves/__qsf003b.html (last accessed: 15 Nov 2014)

Lehota, J., Horváth, Á. \& RÁcz, G. (2014): The effect of sustainability on the information search behaviour of Hungarian consumers through the practice of food purchasing. Acta Alimentaria, 43, 437-443.

Lugasi, A., HoracseK, M. \& Martos, É. (2010): Étrend-kiegészitők a hazai piacon: a termékek forgalmazását, összetételét meghatározó jogszabályi elöírások (Food supplements on the Hungarian market: nutritional assessment of the ingredients, risks and benefits, the role of food supplements in human nutrition). Orv. Hetil., 151(48), 1964-1975

MóczÁr, Cs. \& RuRIK, I. (2015): Comparison of cardiovascular screening methods and mortality data among Hungarian primary care population: preliminary results of the first government-financed managed care program. Zdrav. Varst., 54(3), 154-160.

NutRICOMP LTD (2013): Available at http://www.nutricomp.hu (last accessed 07 Jul 2015)

Parker-Pope, T. (2007): A high price for healthy food. Available at http://well.blogs.nytimes. com/2007/12/05/a-high-price-for-healthy-food/? r= 0 (last accessed 30 Nov. 2015)

Peirson, L., Douketis, J., Ciliska, D., Fitzpatrick-Lewis, D., Ali, M.U. \& Raina, P. (2014): Prevention of overweight and obesity in adult populations: a systematic review. CMAJ. Open., 2(4), 268-272.

Powell, L.M., Chriqui, J.F., Khan, T., WadA, R. \& Chaloupka, F.J. (2013): Assessing the potential effectiveness of food and beverage taxes and subsidies for improving public health: a systematic review of prices, demand and body weight outcomes. Obes. Rev., 14(2), 110-128.

Sarkadi Nagy, E., Bakacs, M., Illés, E., Zentai, A., Lugasi, A. \& Martos, E. (2012): Országos Táplálkozás és Tápláltsági Állapot Vizsgálat - OTÁP 2009. II. A magyar lakosság energia- és makrotápanyag-bevitele (Hungarian Diet and Nutritional Status Survey - the OTAP 2009 study.) II. Energy and macronutrient intake of the Hungarian population. Orv. Hetil., 153(27), 1057-1067.

Story, M., Kaphingst, K.M., Robinson-O’Brien, R. \& Glanz, K. (2007): Creating healthy food and eating environments: Policy and environmental approaches. Ann. Rev. Publ. Health, 29, 253-272.

USDA (2010): Dietary guidelines for Americans. U.S. Department of Agriculture,. Department of Health \& Human Services, Available at http://www.dietaryguidelines.gov (last accessed 25 Apr 2013)

Vetter, M.L., Amaro, A. \& Volger, S. (2014): Nutritional management of type 2 diabetes mellitus and obesity and pharmacologic therapies to facilitate weight loss. Postgrad. Med., 126(1), 139-152. 